\title{
Mapping in unstructured natural environment: a sensor fusion framework for wearable sensor suites
}

\author{
Georges Chahine ${ }^{1}\left(\mathbb{D}^{\circ} \cdot\right.$ Maxime Vaidis $^{2} \cdot$ François Pomerleau $^{2} \cdot$ Cédric Pradalier $^{3}$
}

Received: 31 October 2020 / Accepted: 3 April 2021

Published online: 21 April 2021

(c) The Author(s) 2021 OPEN

\begin{abstract}
We present a generalized mapping framework that can withstand the challenges incurred by working in unstructured outdoor environments, such as a snowy forest. The proposed method takes advantage of a sensor fusion scheme, where sensors such as cameras and lidars are used in order to reconstruct the surrounding natural environment. Although mapping techniques such as SLAM and ICP cannot themselves properly handle the complexity of natural scenes, they do have the potential to contribute to the global solution in a proposed sensor fusion scheme, based on a factor graph architecture. In this paper, we propose an innovative map registration scheme for visual maps, and show how it can improve the reconstruction quality after data fusion. We also analyze the behavior and sensitivity of factor graphs to uncertainties, by comparing the residual error with different parameter combinations such as variances, using an exhaustive grid search with ground truth comparison. Finally, we suggest an ICP-inferred loop closure, capable of compensating position and attitude drift. The experiments are carried out by recording in a snowy forest using a wearable sensor suite. In the experiments, ground truth was acquired using a millimeter-accurate total station. The proposed framework is shown to be robust and likewise capable of providing estimates that are otherwise unattainable using classic techniques, such as visual SLAM and ICP for lasers. Finally, a visible improvement in the map reconstruction quality is shown, and the proposed framework achieves a translation error of $0.36 \mathrm{~m}$.
\end{abstract}

Keywords Mapping · Natural environment · Sensor fusion - Wearable robots

\section{Introduction}

Autonomous robotic systems require a representation of their environment, such as a map, which is used to achieve specific objectives. For example, these maps are used to develop autonomous movement strategies (e.g., path planning, obstacle avoidance) or to perform specific tasks such as forest inventory [19]. In some cases, the map is the purpose of the mission. For example, data obtained after processing a map can be used to extract features such as tree diameters [23].
Several mapping solutions exist, yet they all depend on the environment in which they are evaluated, and on the desired task which might also be affected by environmental conditions. [4, 29]. Based on that, the successful execution of a given mapping technique will have to take into account the environmental challenges that might cause it to fail. In this study, we will focus on the three-dimensional reconstruction of unstructured environments such as a forest, during challenging meteorological conditions. Such environments are a challenge for mapping algorithms such as

Georges Chahine, gchahine@gatech.edu; Maxime Vaidis, maxime.vaidis@norlab.ulaval.ca; François Pomerleau, francois.pomerleau@norlab.ulaval.ca; Cédric Pradalier, cedric.pradalier@georgiatech-metz.fr |'Georgia Institute of Technology, Atlanta, GA, USA. ${ }^{2}$ Université Laval, Quebec City, QC, Canada. ${ }^{3}$ Georgia Institute of Technology, UMI 2958 GT-CNRS, Georgia Tech Lorraine, Metz, France. 
Simultaneous Localization And Mapping (SLAM), but also for pose estimation of the robotic platform because of the roughness of the terrain. Traditionally, several types of sensors such as cameras and lidars can be used to obtain the pose of a robot.

Cameras are of a particular interest for applications involving robots, as they are considered sensors that can be used for localization and mapping i.e., SLAM. Monocular cameras are particularly versatile, lightweight and comparatively cheaper than lidars, making them suitable especially for wearable sensor suites. Visual SLAM techniques allow the generation of pose and map estimates from a sequence of moving images. The booming of learning-based techniques meant that cameras can also significantly contribute to the decision-making process in artificial intelligence applications.

When extrinsics are available, cameras have the ability to complement lidars, given that they often include color or semantic data, that can be projected onto the lidar point cloud. Unfortunately in some environments, extracting robust features from camera images can be challenging, such as when working with featureless, snow covered landscapes. As a result, it becomes difficult to use a method purely based on visual odometry or vision-based SLAM.

As a result, convenience implies the use of other sensors such as lidars that perform better under visually challenging constraints. Unlike cameras, lidars provide high-precision point clouds and are capable of producing dense maps within their range. Using the Iterative Closest Point (ICP) algorithm [2], lidar scans are incrementally matched to build a three dimensional map.

The ICP algorithm iteratively compares two point clouds in order to find the rigid transformation that minimizes the alignment error between them. Although this method ensures local consistency, a global drift appears over time [20]. In case the trajectory does not loop, the drift cannot be corrected using a loop closure, hence it is necessary to use other techniques to refine the pose of the robotic platform. These drifts can be also attenuated by SLAM methods that use optimization graphs [5], or by constraining the position of the data acquisition system using Global Position System (GPS) priors.

Some research has used deep learning with range sensors in order to decrease the processing time during map registration such as [25]. These methods have the advantage of not adhering to the rigid motion assumption to compute the pose of the system. Other point cloud registration techniques include the Normal Distribution Transform (NDT) algorithm [3], although favorable results require a lidar with at least 32 beams [16]. The affordability and increasing popularity of 3D lidars have driven an interest in 3D point cloud descriptors such as 3DSmoothNet [11] and in correspondence alignment techniques such as TEASER++ [28].

The state-of-the art in SLAM has so far failed to address the challenges encountered in unstructured natural environments [6]. Further, visual SLAM techniques lack both scale and the density seen in maps generated using 3D lidars. In a previous work, we surveyed the performance of monocular visual SLAM algorithms in natural environments [6], and found that Direct Sparse Odometry (DSO) [10] has a performance edge when it comes to natural environment applications.

We call data fusion any scheme or approach that takes advantage of multiple inputs from sensors such as GPS, Inertial Measurement Unit (IMU), and possibly some kind of odometry including, wheel, visual and ICP-based. The purpose of sensor fusion is to obtain a refined estimate of a quantity. In this paper, it is the 6 Degrees of Freedom (DoF) pose, which is otherwise unattainable using an individual sensor [13]. For the purpose of fusing pose estimates, factor graphs are capable of integrating different estimates such as odometry and GPS readings i.e., geometric constraints [17]. The trajectory to be estimated is discretized into several points of reference along it, which we call nodes. These nodes represent the robot 6 DOF pose and they are interconnected using geometric constraints, as well as priors that reflect absolute knowledge on the pose of a robotic platform. What follows is a smoothing and optimization step to refine the pose estimate at each node [9]. Factor graphs are therefore powerful tools for sensor fusion since constraints and priors can be inferred from multiple sources.

The goal of this paper is to evaluate and adapt available techniques such as factor graphs, ICP and scale-lacking monocular visual odometry for the purpose of creating visual representations of natural environments, using visual and range sensors such as cameras and lidars, respectively. We show how we can recover the scale in the context of a factor graph, improve the reconstructed visual map after sensor fusion and use visual odometry to further refine pose estimates.

We highlight the sensitivity of factor graphs to uncertainties and determine unknown parameters such as uncertainties on visual odometry, by running an exhaustive grid search to determine the best parameter combination. We also explain how noisy visual map reconstruction can be even after pose optimization, and suggest as a solution a piecewise reconstruction and alignment scheme. The suggested framework includes a loop closure step, inferred using ICP in revisited geographic areas. In this paper, we also evaluate the suitability of the state-of-theart in correspondence alignment for mapping in natural environment. We underline performance of ICP and its suitability for lidar scan matching on slowly moving robotic 
platforms such as wearable sensor suites. Our approach was tested by recording data in forest during sunny and snowy weather, with the sensor suite presented in Fig. 2 . In Sect. 4, the results were compared to a ground truth given by a theodolite in order to have an accurate assessment of the pose given by the factor graph.

\section{Related work}

The generation of 3D maps from a moving platform is an evolving field of study, due to the increasing availability and affordability of exteroceptive sensors such as cameras and lidars. Unfortunately, the majority of available techniques have either focused on indoor or structured outdoor scenes, with few of these methods such as [6] and [10] for visual methods along with [1] for laser-based methods having partial success in unstructured outdoor scenes, and in particular the reconstruction of trees. In the context of a natural environment, the state-of-theart does not provide specialized solutions for 3D reconstruction, yet recent works such as [27] and [7] are starting to address some of the challenges associated with 3D reconstruction of natural organic structures. The task of trees crown reconstruction remains unsolved due to the variability of trees phenotype, with some research focused on CAD model replacement [8] or radius estimation from flying laser measurements [15]. Additionally, works such as [26] and [27] show that it might be easier to model tree structure such as a trunk, branches and twigs when they are exposed and observable, such as during winter when the trees are bare. Another challenge is meshing the point clouds to create surfaces that are suitable for autonomous robot navigation such as path planning, with few libraries such as [21] and [24] providing useful meshing tools. The topological representation of a point cloud is, however, open-ended and in many cases specific to the application. Nevertheless, some libraries such as [24] provide hole detection and filling tools, and most point cloud processing libraries such as [21] and [24] provide some form of plane fitting for surface estimation, usually using RANSAC or marching cube techniques for surface fitting. HDL Graph SLAM [16] presents a graph-based sensor fusion approach to mapping, capable of integrating GPS, IMU and laser scanners. The use of the word "HDL" references to the authors' experiments with the velodyne HDL32e laser. HDL SLAM does not support the integration of visual odometry and when testing the method, it has been noticed that there is no scheme for dense reconstruction in between graph nodes.

In a recent work [7], we presented a real-time 2D lasersupported visual tracking solution for natural environment, dependent on a camera feed and a sparse disparity image generated by projecting a $2 \mathrm{D}$ laser onto the corresponding image. However, laser-supported visual odometry assumes an overlap between the lidar field of view and that of the camera. In addition that, it does not support the integration of multiple cameras or inertial measurements.

\section{Framework}

The proposed framework, shown in Fig. 1, is a sensor fusion scheme based on a factor graph and in particular the implementation of Dellaert et al. [9]. The data is assumed to be time synchronized, as detailed in the implementation presented in Sect. 4. For the purpose of this section, the system is assumed to have, at least, one monocular camera, one 3D laser, an IMU and a GPS receiver. An approximation of the camera intrinsics and the various extrinsics between sensors is assumed to be known as well. The 3D laser can be replaced with a 2D laser, as long as that the field of view of the laser intersects with that of at least 1 camera. Unfortunately, laser-based SLAM is unsuitable for vertically installed $2 \mathrm{D}$ lasers such as in Fig. 2a, given the lack of overlap in between measurements. In this paper, the use of 'global frame' refers to the Universal Transverse Mercator (UTM) frame and in particular the North-East-Down (NED) convention, representing $x$, $y$ and $z$ respectively.

\subsection{Data synchronisation}

Different sensors exhibit different latencies that cannot be precisely measured or compensated. Further, cameras suffer additional delays such as image integration time (shutter length), and are susceptible to delays during data transmission, due to high bandwidth requirements in high frequency transmission. Consequently, asynchronous data collection does not guarantee data association and temporal synchronization, as highlighted in [12] and [14]. To solve the latter problem, the suggested framework takes advantage of a synchronized physical trigger.

\subsection{Factor graph}

We define a factor graph node distancing parameter $d$, that constrains the number of nodes in a factor graph. For instance, between two consecutive nodes, the robotic platform would have traveled at least $d$ meters along the trajectory. The distancing parameter cannot be inferred from visual odometry due to lack of scale. Instead, other solutions such as IMU-aided GPS, laserbased ICP (for 3D lasers) or laser-aided visual odometry (for 2D lasers) can be used to determine the node distancing parameter. In this paper, we construct our nodes 
Fig. 1 Flow chart of the proposed framework, including the sensors used during experimentation
Fig. 2 Implementation of the design shown in Fig. 3

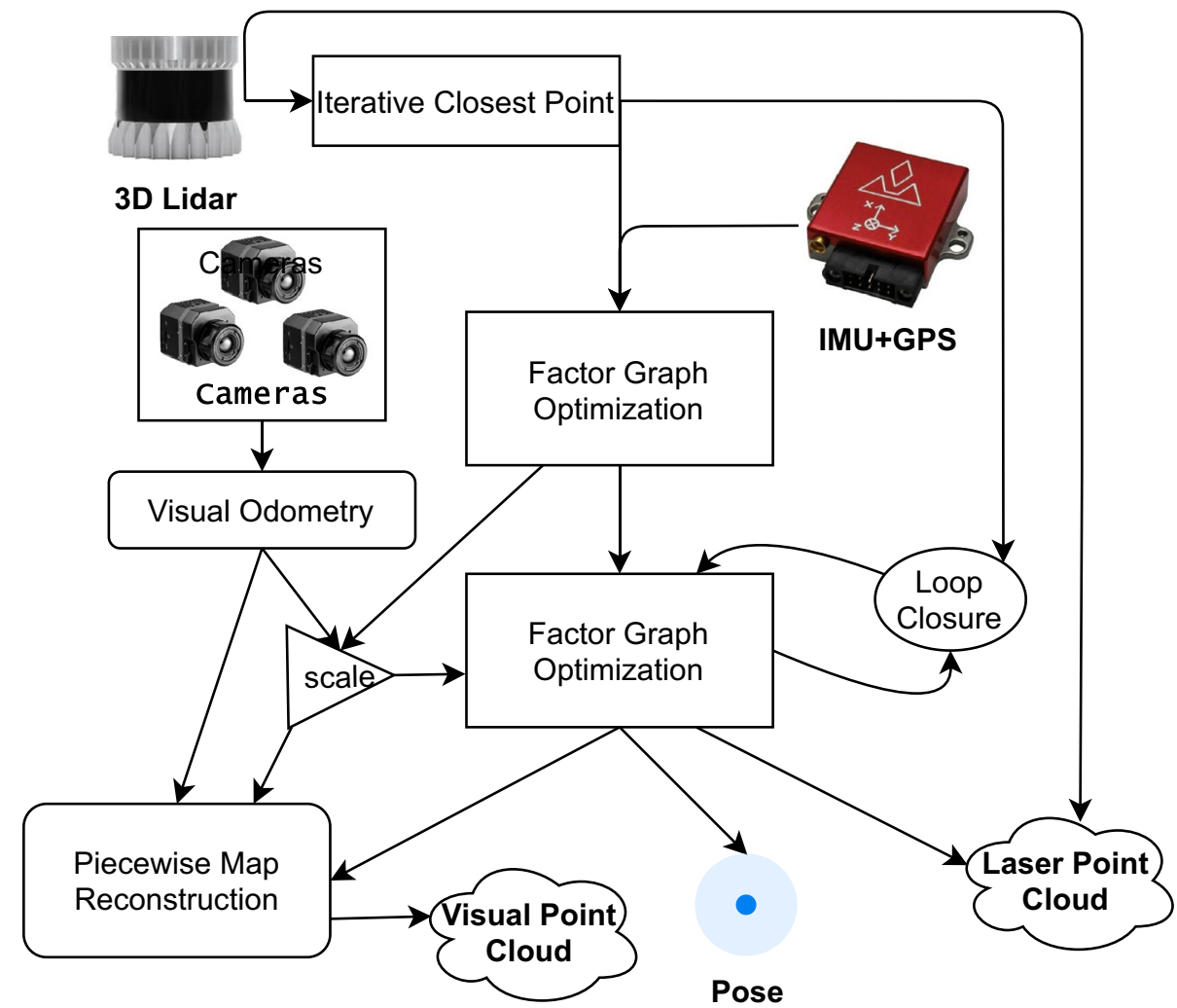

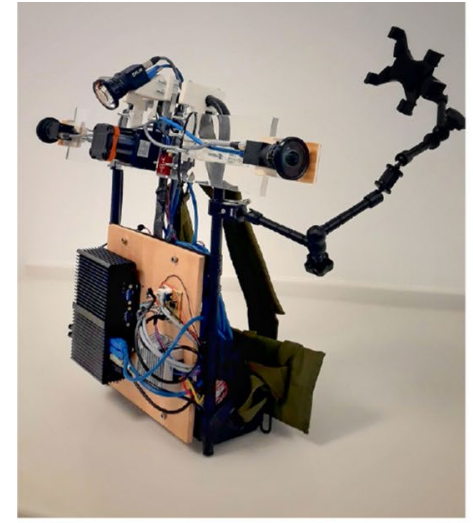

(a) The backpack used for data acquisition, an execution of the design seen in Figure $3 \mathrm{a}$.

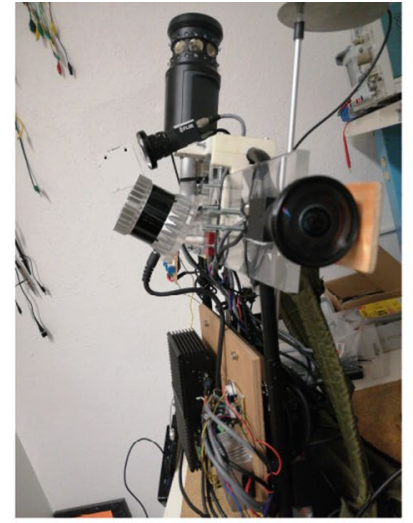

(b) The backpack with a 3D laser shown in Figure $3 \mathrm{~b}$, and the theodolite prism.

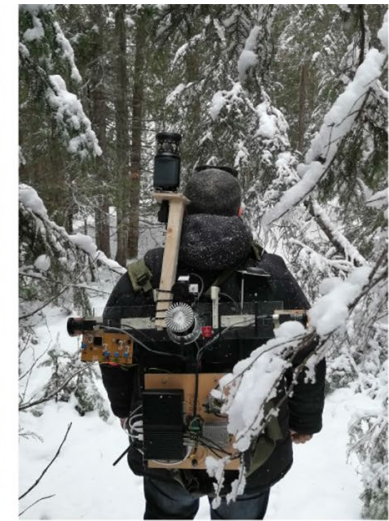

(c) Data acquisition in a snowy forest in Quebec, Canada. by downsampling the input GPS-IMU data, using a distance-based downsampling scheme, i.e., discarding all but the first measurement, each time the system moves by $d$ meters. The latter scheme therefore accounts for occasional movement pauses while traversing rough terrain despite non-interrupted data recording, such as in human-carried sensor suites.

Let $d_{\text {gps }}$ represent the sparsity of GPS prior constraints, according to which the GPS measurements are distributed over the factor graph nodes. In other words, we constrain our pose with GPS measurements every $d_{\text {gps }}$ nodes i.e., $d_{g p s} * d$ meters. We now construct a factor graph with:

- A GPS prior every $d_{\text {gps }}$ nodes.

- An attitude prior inferred from the INS, which describes the attitude of the IMU with respect to the global frame (UTM-NED) with bias compensation using the GPS direction of motion propagation. In the experimental section, bias compensation is computed internally inside the INS sensor. 
- Multiple odometry readings, either from laser-based ICP and/or laser-supported visual odometry. A robotic platform with wheel odometry can also be used as an alternative. In this paper, we constrain our system using ICP odometry and scaled visual odometry.

\subsection{Scale recovery and interpolation}

The lack of scale in visual odometry measurements prevents the insertion of scaleless constraints into the factor graph. Instead, we need to scale these measurements either using GPS or a less noisy estimate from a preliminary factor graph optimization, given the availability of ICP odometry. Using the factor graph defined above, a preliminary factor graph optimization using GPS and attitude priors, as well as ICP odometry is performed and the resulting pose is used to scale visual maps, as shown in Fig. 4.

The scale for a visual odometry constraint at a given factor graph node $n$ is estimated by simply accumulating the traveled distance along the trajectory from visual odometry, over the traveled distance inferred from the optimized pose i.e., $s_{n}=D_{v o} / D_{o p t}$, where $s_{n}$ is the scale for a visual SLAM constraint, with $D_{\text {vo }}$ and $D_{\text {opt }}$ representing the cumulative traveled distance over the last $100 \mathrm{~m}$ before reaching the current position at node $n . D_{v o}$ and $D_{o p t}$ are inferred from visual odometry and the preliminary factor graph optimization, respectively.

For each camera, we then interconnect nodes with scaled odometry constraints, followed by another optimization run to refine the pose and consequently the estimated scale. Additionally, three-dimensional reconstruction of the maps requires the recovery of the $6 \mathrm{DOF}$ pose at the original rate. This is also important for cameras due to the keyframe-based nature of many visual algorithms, and therefore there is a need to interpolate the optimized 6-DOF pose. Using timestamp queries with the optimized pose from factor graph, we linearly interpolate the translation component of the pose. As for the rotation part, we use quaternions Spherical Linear Interpolation (SLERP) [22].

\subsection{Point cloud registration}

Point cloud registration is dependent on the context in which the data was captured and the sensor used. For instance, vertically installed 2D lasers cannot be registered using ICP, due to lack of overlap in between scans. In this subsection, we exploit the various solutions to point cloud registration, for both $3 \mathrm{D}$ lasers and cameras.

\subsubsection{Merging of visual maps}

Maps captured by most Visual SLAM algorithms work on the basis of keyframes, which do not necessarily overlap, hence ruling out ICP as a solution to keyframe registration. Unlike lasers, cameras can see far away objects that might show up in a keyframe even after the sensor suite has long left the vicinity of the object. Without breaking keyframe integrity, this results in a noisy, coarse reconstruction when attempting to reconstruct the map by simply transforming the keyframes using the optimized pose from factor graph, or any other pose but the original pose from visual odometry. We therefore suggest a hybrid approach for point cloud registration consisting of local reconstruction of the map using visual odometry, with ICP smoothing for the piecewise alignment of map fragments.

For a set of visual SLAM keyframes $S_{i}$ captured along a portion of the trajectory, we transform any point $P_{i j}$ in the sensor frame to the global frame using $P_{z j}=T_{i j} T_{e x t} P_{i j}$, where:

$T_{i j}$ represents the forward transformation from the base frame to the global frame, $T_{\text {ext }}$ is the extrinsic transformation, also known as the sensor to base transformation, $P_{z j}$ is the projected point in the global frame, and $j$ represents the keyframe number, with $j=1$ referring to the first keyframe.

Let $\mathcal{M}_{c}$ and $\mathcal{M}$, be 2 preliminary point clouds used to construct a refined point cloud $\mathcal{M}_{f}$. All three point clouds, shown in Fig. 5, represent the same scene but are differently constructed.

$\mathcal{M}_{c}$ is a coarse point cloud constructed by simply projecting visual SLAM keyframes using the corresponding optimized pose from factor graph. In other words, $\mathcal{M}_{c}$ is constructed by inferring $T_{i j}$ from the factor graph optimized pose.

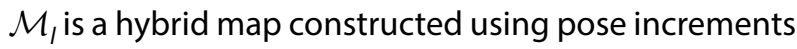
from sensor original data i.e., visual SLAM. The latter pose increments build on sparse factor graph poses, serving as anchors distributed along the optimized trajectory. What follows is the derivation needed to reconstruct a point cloud $\mathcal{M}_{\text {. }}$

At the beginning of every set of keyframes, we set $T_{i, j=1}$ $=T_{o_{k^{\prime}}}$ where $T_{o_{k}}$ represents the optimized pose from factor graph at the corresponding node $k$. This has an anchoring effect and prevents the map from significantly drifting away from the associated pose. We now derive the forward transformation that locally projects the points to the global frame, i.e., $T_{i j}$, dropping the subscript $i$ for brevity with $j \neq 1$ :

$T_{j}=T_{j-1} T_{v o_{j-1}}^{-1} T_{v o_{j}}$

where $T_{v o}$ represents the sensor to world transformation, from visual odometry.

Setting $T_{i 1}=T_{o_{k}}$ on the first keyframe of every set implies a misalignment of map fragments, due to the resetting effect upon which Eq. 1 builds up subsequent pose 
increments. To solve this, we correct the point cloud $\mathcal{M}_{1}$ to obtain a point cloud $\mathcal{M}_{f}$ by aligning, using ICP, the points in a given set $S_{i}$ with the corresponding points in the coarse point cloud $\mathcal{M}_{c}$.

\subsubsection{Merging of range maps}

Range scans obtained from 3D lasers overlap and therefore are suitable for ICP-based solutions. In this paper, a hybrid approach for map registration identical to the method presented in Sect. 3.4.1 is used by replacing visual odometry by an ICP mapper that takes advantage of an interpolated pose prior from factor graph. In other words, we register $\mathcal{M}$, using ICP in-between factor graph nodes, and $\mathcal{M}_{c}$ using the interpolated pose from factor graph.

\subsection{Exhaustive parameter search}

An exhaustive search for parameters is a brute force technique consisting of trying all possible values of a parameter, while looking for desired changes in a system dependent on the said parameter. Feedback on the search process is dependent on the application and the system at hand. Exhaustive search techniques are especially useful when a priori knowledge on the value of a parameter is poor or unavailable.

The proposed parameter search scheme is an exhaustive search of factor graph uncertainties as well as other parameters that might affect the outcome. For instance, we noticed that varying $d_{g p s}$ has a considerable effect on the outcome, and therefore $d_{g p s}$ was included in the parameter search scheme along with the uncertainties. The proposed exhaustive search implies trying all parameter combinations in a constrained range of values, followed by a factor graph optimization step. We then compute the error feedback and log all the parameters with the associated error value.

Setting the covariance matrices for a factor graph can be challenging: unlike sensors that come with a datasheet, laser-based ICP or visual odometry do not have a defined uncertainty. Although the parameters can be manually fixed, a better estimation can be achieved through an exhaustive parameter search scheme, which runs through a constrained range of values to determine the covariances associated with visual odometry, ICP-generated pose, GPS and IMU measurements as well as the factor graph parameter $d_{\text {gps }}$.

Subsequently, parameter search is performed by logging different parameter combinations against alignment error with the reference (i.e., ground truth) trajectory. Unfortunately, an exhaustive parameter search is a computationally expensive task, and in this context assumes the availability of ground truth data. Further, uncertainties for different measurements are dependent on the sensor as well as the environment around it. For instance, laser measurements depends on the surface material upon which laser beams reflect, as well as climatic conditions. For the purpose of the exhaustive parameter search, the bulk of the uncertainty is assumed to be interoceptive to the sensor or method used to generate the measurements. The latter assumption hypothetically allows the generalization of the parameters for a sensory platform, disregarding exteroceptive stimuli. Further discussions in Sect. 4 show that the parameters we find for a given survey remain valid for other surveys that might not include the ground truth required to run the exhaustive parameter search.

\subsection{Loop closure}

Position and attitude drift is expected with most incremental sensor systems. For an outdoor system such as the one presented in this paper, both visual and laser-based measurements accumulate errors, the latter constrained by IMU-aided GPS priors, in the context of a factor graph. In this section, we suggest a two-step loop closing scheme; the first step consisting of a correspondence match generation function followed by a laser-based, ICP-inferred geometric constraint.

\subsubsection{Correspondence generation}

The underlying assumption for correspondence generation is that translational drift, constrained by GPS priors, is well below the laser range threshold, currently between 30 and $50 \mathrm{~m}$ for commercially available 3D lasers. Given a factor graph with $n$ nodes, with the first node being $N_{1}$ and the last node being $N_{n}$. We generate the $n \times n$ correspondence map $\mathcal{C}$, such that $\mathcal{C}_{(i, j)}=1$ if the laser-generated maps associated with neighbor nodes $N_{i}$ and $N_{j}$ overlap, and $\mathcal{C}_{(i, j)}=0$ everywhere else. To check for corresponding nodes, the fifty nearest neighbors for a node $N$ are tested against two distance constraints. The first constraint is a proximity parameter, typically between 5 and $15 \mathrm{~m}$ that guarantees map overlap. The second constraint is an elapsed distance over the trajectory constraint typically between 15 and $30 \mathrm{~m}$. Every node $N_{i}$ is associated with a distance $D_{i}$, which measures the elapsed distance over the trajectory from $N_{1}, N_{2}, \ldots$ to $N_{i}$. The second constraint guarantees that the nearest neighbor lies in a revisited space, after the sensor suite has initially left the area.

\subsubsection{Geometric constraint}

We propose an ICP-based geometric constraint, using the correspondence map found in Sect. 3.6.1. Owing to the 
Fig. 3 All fixtures were 3D printed or laser-cut according to a pre-designed CAD model, so that to minimize geometric projection error in between components. The instruments are mounted on a rigid acrylic plate, the latter affixed onto the metal frame of a backpack

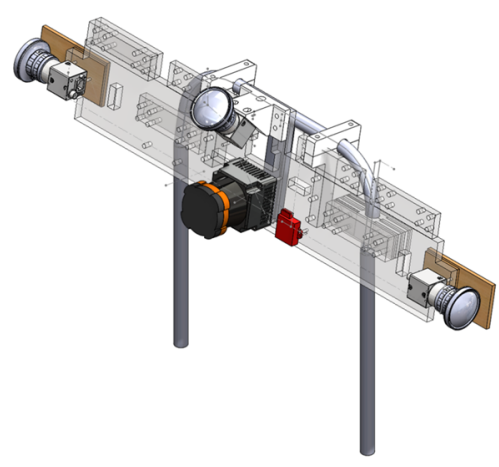

(a) The backpack CAD model, showing a 2D Hokuyo laser (center), an Inertial Navigation System (INS) here in red, and three cameras: two on the sides and a center camera.

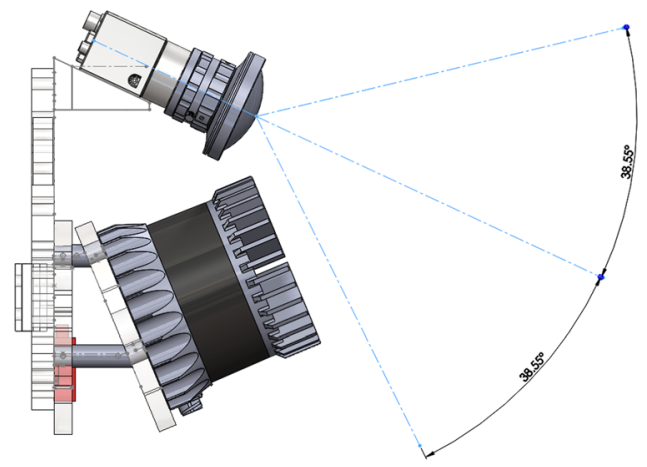

(b) Backpack CAD model, showing the Ouster16 lidar model (bottom) on a custom-made mount to avoid interference with the field of view of the camera (top).

Fig. 4 Factor graph flow chart

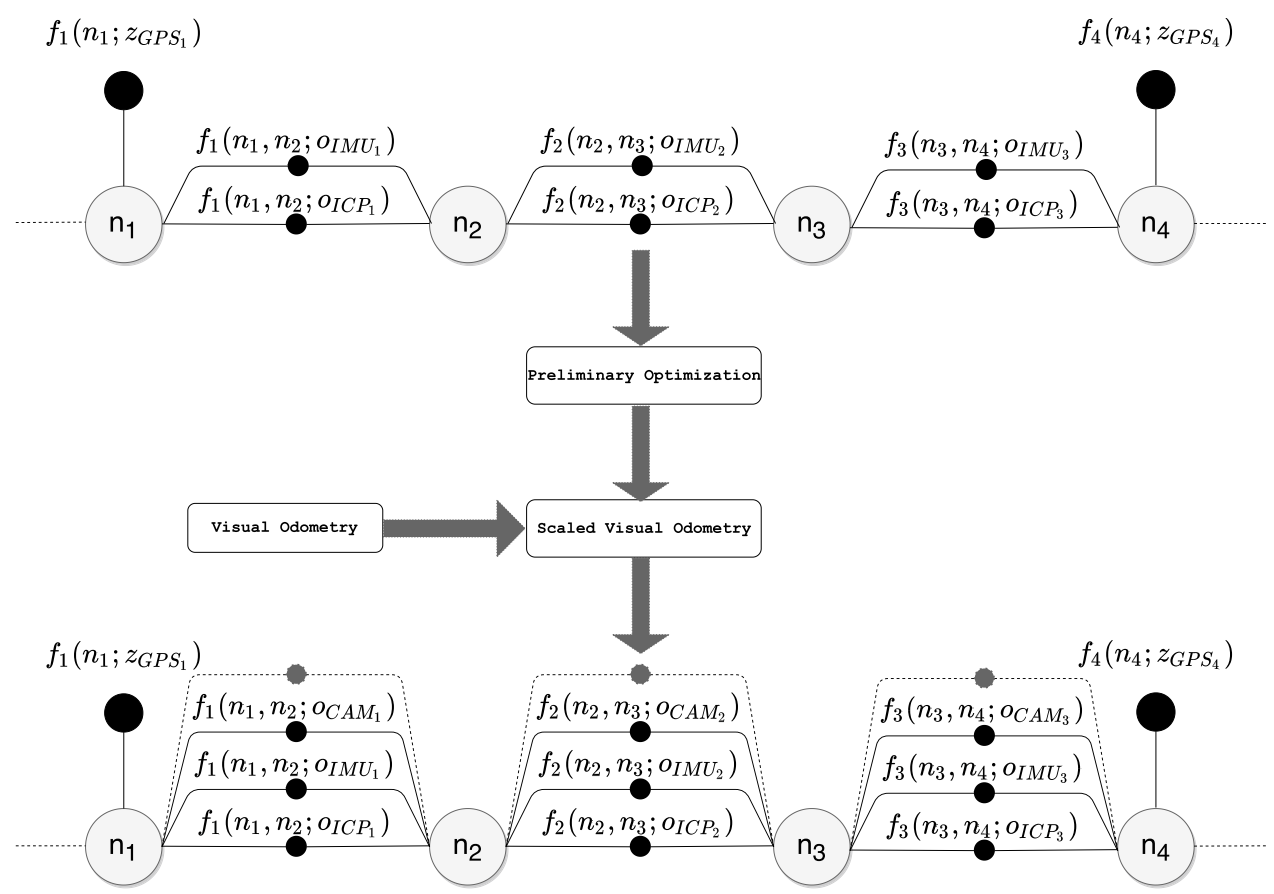

constrained nature of the field of view and namely the opening angle of 3D lasers, the point clouds associated with two corresponding nodes i.e., just two laser scans, are not guaranteed to significantly overlap. As a solution to that, using the optimized pose from the factor graph, we locally reconstruct the laser map observed from consecutive factor graph nodes $N_{1}, \ldots, N_{k}$, using interpolated factor graph poses $P_{1}, \ldots, P_{k}$ to create sub-maps. A matching sub-map is subsequently found using the correspondence $\operatorname{map} \mathcal{C}$. A series of nodes and their associated maps form a sub-map if and only if every node in the series associate with at least a single correspondence in $\mathcal{C}$, and that no significant gaps exist in between consecutive scans to guarantee map continuity. Further, we define the map size limit such using the length of the trajectory $\left(P_{k}-P_{1}\right)<T h_{l c}$, where $T h_{l c}$ is the map length limit over the trajectory typically between 10 and $40 \mathrm{~m}$, over which a new match pair is initialized. Finally, for every match pair, we infer the geometric transformation using ICP and add the inferred constraint to the factor graph.

\section{Experiments}

The dataset used in this paper is composed of a forest environment, in the area surrounding Montmorency Forest in Quebec, Canada, shown in Fig. 6. A secondary survey was also captured in an open field next to Université Laval. 


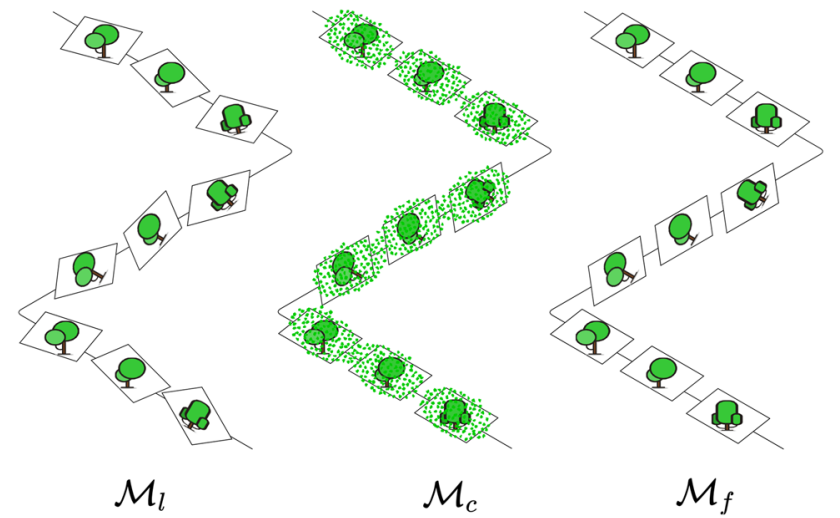

Fig. 5 Schematic showing the 2 intermediate maps $\mathcal{M}_{c}, \mathcal{M}_{1}$ and the final map $\mathcal{M}_{f}$. Snapshots of $\mathcal{M}_{c}$ and $\mathcal{M}_{f}$ are shown in Fig. 11

Data were captured using the sensor suite fitted on the backpack shown in Fig. 2a. As shown in Fig. 3, the data acquisition system has been designed to include three 2.0 megapixels RGB cameras from FLIR, a 16-beam Ouster-16 lidar from Ouster, and the VN-200 INS from Vectornav, the latter consisting of an IMU and a GPS. The system is physically synchronized using a laser-generated trigger, that can be downsampled using an on-board frequency divider circuit. Finally, the data is recorded on the on-board computer. For the remainder of this paper, the term 'forest survey' refers to the dataset captured in the snowy forest shown in Fig. 6b.

The versatility of a backpack allows survey recording in narrow or difficult to navigate regions, as shown in Fig. 2c. Further, designing sensor fixtures and having them rigidly affixed to the backpack frame, means that sensor extrinsics can be calculated by using measurements inferred from the design model. For cameras, this includes taking into account the location of the image plane inside the camera hull, the latter provided by the camera manufacturer datasheet. Further, we used a Trimble S7 theodolite to obtain a translational ground truth. By affixing a theodolite prism onto the backpack as shown in Figs. $2 \mathrm{~b}$ and $6 \mathrm{a}$, we were able to have an accuracy to the millimetre of its trajectory.

The exhaustive parameter search was performed by defining four lists $L_{1}, L_{2}, L_{3}, L_{4}$ that contain, respectively, a range of uncertainties for GPS, visual odometry, and ICP measurements as well as the sparsity parameter for GPS constraints $d_{g p s}$. The lists of uncertainties are designed to be inclusive of all logical potential values:

- The list of GPS uncertainties $L_{1}$ ranges between 1 and 19 , discretized with steps of $1.5 \mathrm{~m}$, for a total of 13 possible parameters.

- The list of visual odometry uncertainties $L_{2}$ ranges between 1 and 16, discretized with steps of $1.5 \mathrm{~m}$, for a total of 11 possible parameters.

- The list of sparsity constraints $L_{3}$ ranges between 1 and 30 , discretized with steps of 1 node, for a total of 30 possible parameters.

- The list of ICP uncertainties $L_{4}$ ranges between 1 and 25 , discretized with steps of $1.5 \mathrm{~m}$, for a total of 17 possible parameters.

In practice, with 4 parameters we have a $O\left(\prod_{i=1}^{4} n_{i}\right)$ complexity, where $n_{i}$ is the number of elements in a list $L_{i}$. The exhaustive search for the four parameters took around 60 hours, using an off-the-shelve Intel Core i7 computer with $16 \mathrm{~GB}$ of RAM. What follows is an exhaustive parameter search as presented in Algorithm 1.
Fig. 6 Data acquisition in forestry environment

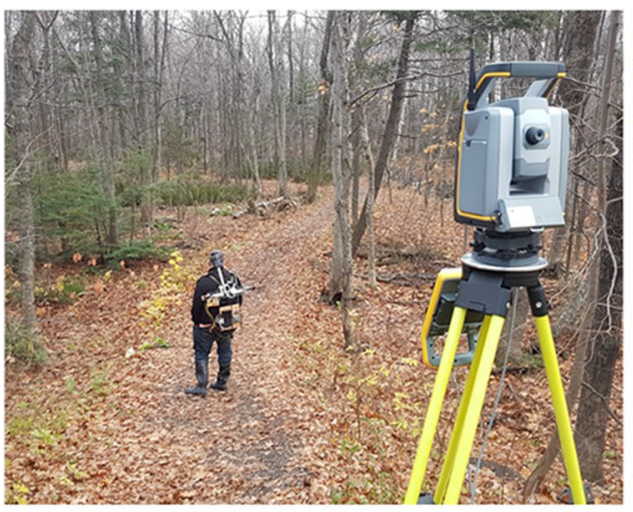

(a) Ground truth recording using the theodolite total station.

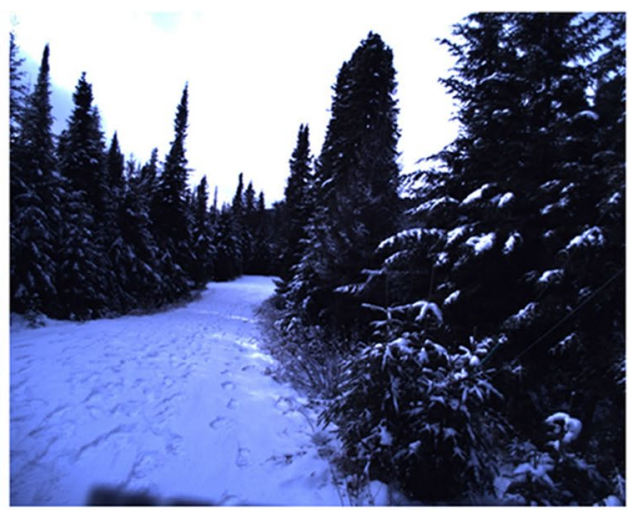

(b) Snapshot from the center, backwardfacing camera on the backpack sensor suite. 


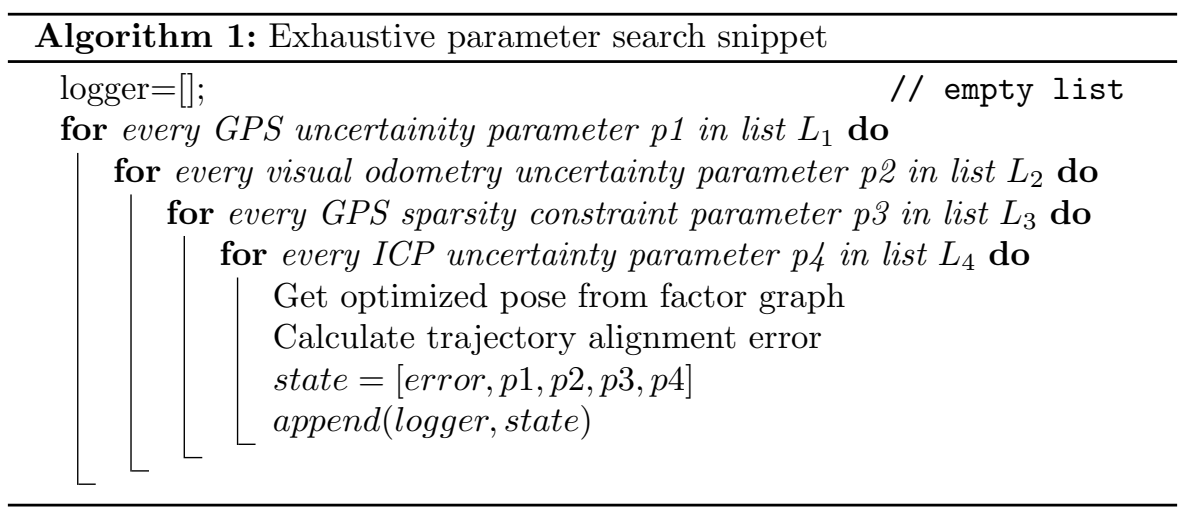

\section{Results}

\subsection{Quantitative analysis}

Figure 7 shows the error variations in a partial parameter search. Still in the same figure, it is shown that the inclusion of visual odometry improves the overall pose estimates, in almost all possible parameter combinations. Moreover, the periodicity in error oscillations suggests that certain parameters tend to be more dominant than others with respect to their contribution to the error term, therefore imposing their own periodicity on that of the error. Based on that and given the mixture of parameters at hand, we generate graphs to highlight the effect of individual parameter variability, as shown in Fig. 8.

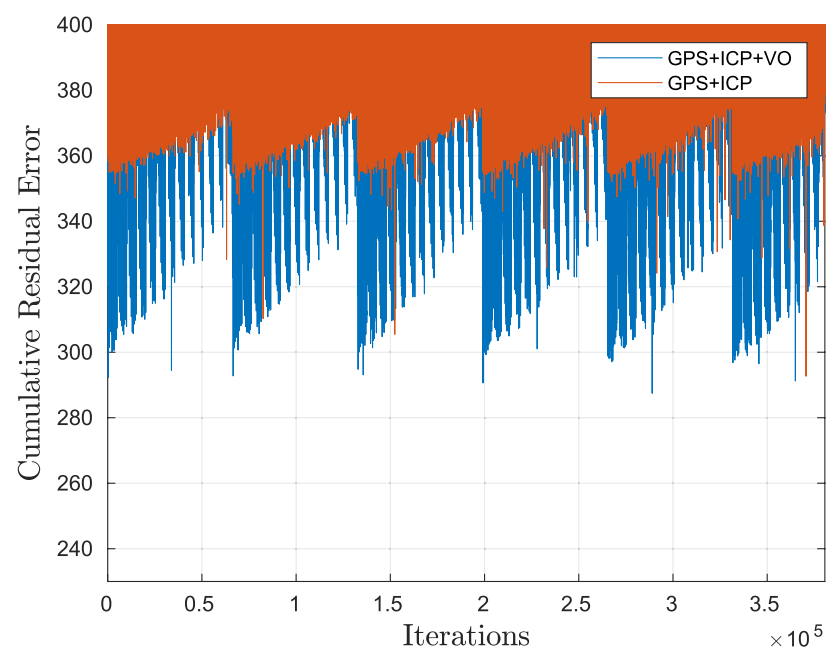

Fig. 7 Cumulative trajectory alignment error, in function of an expanded set of parameter combinations, with and without visual odometry
The sensitivity of factor graphs to the estimated uncertainties, here expressed in variances, is highlighted in Fig. 8, proving the existence of a narrow range of variances, at which the factor graph optimization performs at its best. Further, certain variances such as those associated with GPS measurements show a more forgiving range compared to the parameter associated with visual odometry or the parameter $d_{\text {gps }}$, the latter two parameters seemingly having a considerable effect on the optimization process. Still in the same figure, the standard deviation of the error can influence parameter selection. For example, Fig. $8 \mathrm{~b}$ shows a considerable fluctuation of the error for laser-based ICP odometry, in the range between 1 and $5 \mathrm{~m}$. Therefore, it is convenient to choose the values between 5 and $10 \mathrm{~m}$, as they suggest an improved stability and therefore a higher repeatability. In contrast to the latter, Fig. $8 \mathrm{c}$ suggests that uncertainties attached to GPS measurements do influence the system performance yet less significantly than the other three parameters in the same figure. This is due to the small range in which the error varies for variance values ranging between 1 and 20 $\mathrm{m}$, reinforced by a largely consistent standard deviation all throughout the range of values.

Comparing the optimized pose from factor graph with the ground truth, we generate the Root Mean Square (RMS) error for the translational component. The alignment RMS error, inferred from the residual RMS error associated with the ground truth trajectory alignment, was added to the evaluation since it penalizes for trajectory deviations and rotation inaccuracies. Given that even small deviations from ground truth, as shown in Fig. 9a are carried through the trajectory and globally affect the alignment quality, the associated error is expected to be significantly higher than its translational counterpart. Nevertheless, a perfect alignment is desirable and therefore it is a suitable metric in comparing different methodologies. 


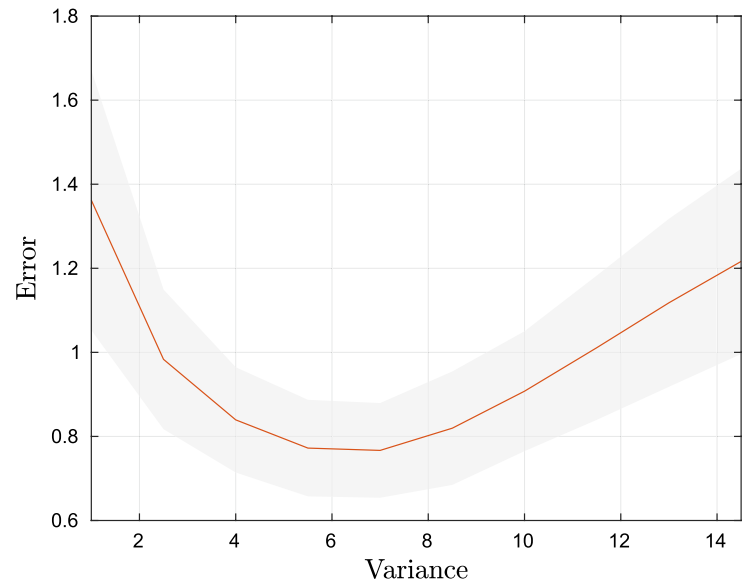

(a) Trajectory error, in function of the variance for scaled visual odometry.

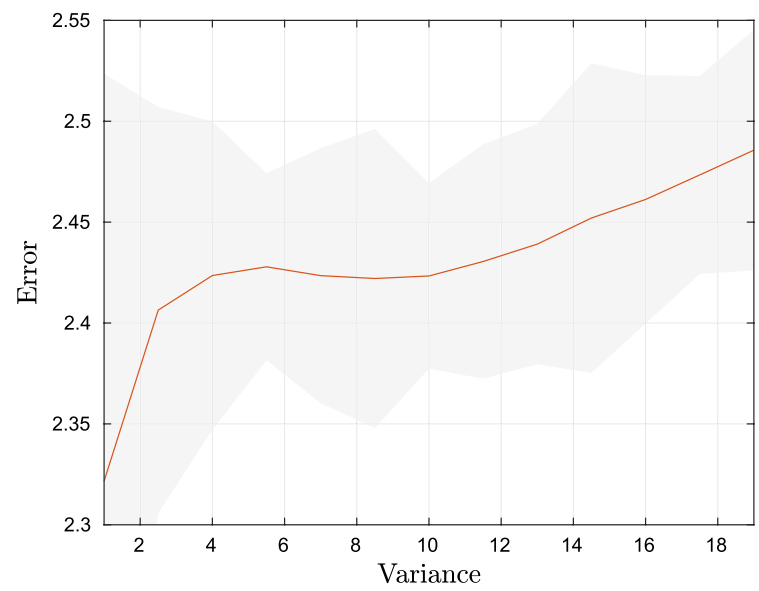

(c) Trajectory error, in function of the variance for GPS measurements.

Fig. 8 Exhaustive parameter search, to evaluate the effect of certain parameters against performance metrics obtained from ground truth. The error values shown above, are the lowest error

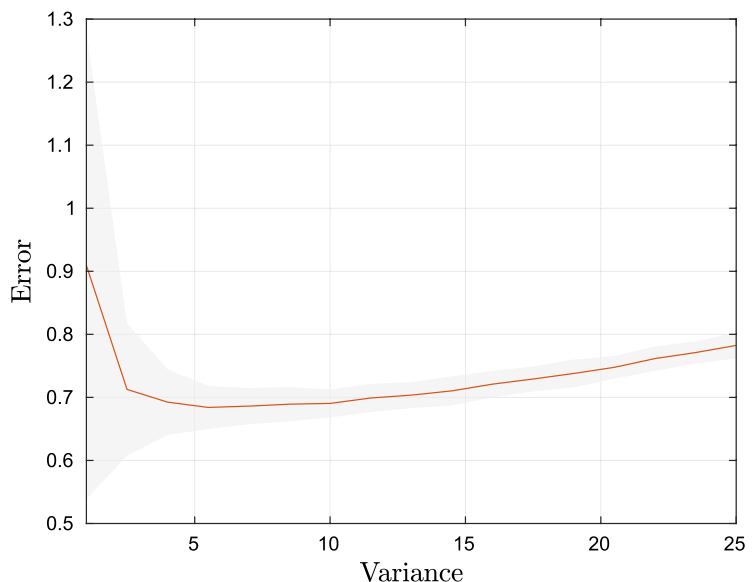

(b) Trajectory error, in function of the variance for laser-based ICP.

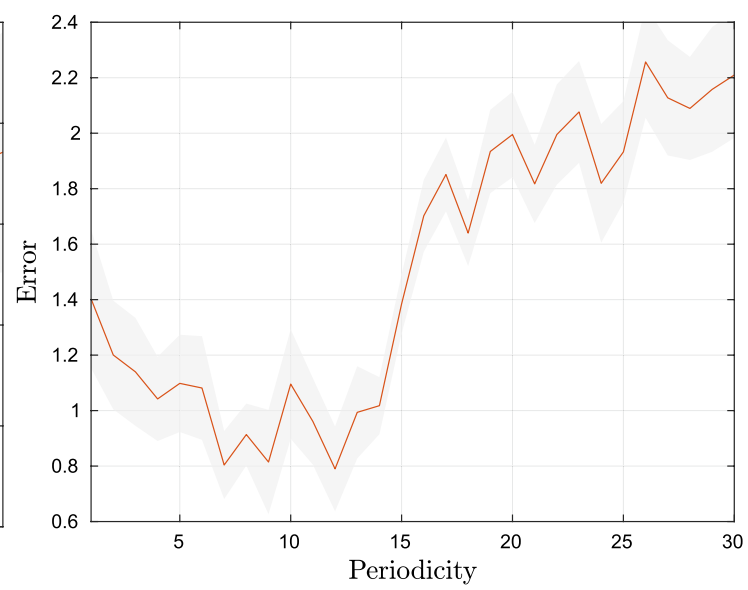

(d) Trajectory error, in function of the periodicity for the parameter $d_{g p s}$.

values (lowest 1\%), obtained by fixing a given parameter and looking at error values against all other parameter combinations. The shaded area represents the standard deviation of the error
Fig. 9 Comparison of trajectories with the ground truth

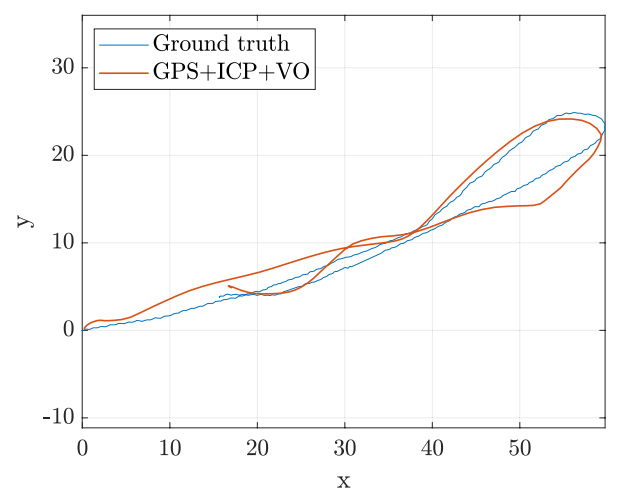

(a) Trajectory alignment of the factor graph optimized pose with the ground truth from the theodolite total station.

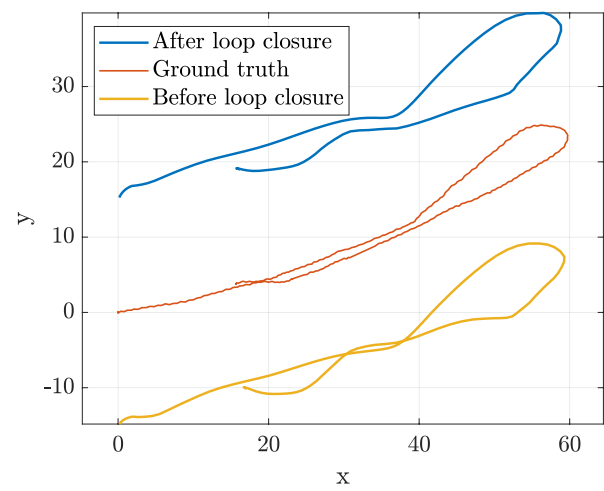

(b) Trajectory drift compensation, before and after loop closure. 
Table 1 Accuracy metrics for the forest dataset and a secondary survey

\begin{tabular}{lll}
\hline Survey & $\begin{array}{l}\text { Translational RMS } \\
(\mathrm{m})\end{array}$ & $\begin{array}{l}\text { Align- } \\
\text { ment } \\
\mathrm{RMS}(\mathrm{m})\end{array}$ \\
\hline Main (forest) & 0.36 & 2.4 \\
Secondary (open field) & 0.45 & 8.29 \\
\hline
\end{tabular}

Table 1 shows the RMS performance for the forest survey, and an additional survey that has been added for the purpose of evaluating the suitability of previously found parameters in other surveys. Still in the same table, a secondary survey run showed the usability of the previously found parameters on another survey, yet with a slight increase in the translational RMS and a 4-fold increase in the alignment RMS. Note that, to the best of the authors' knowledge, no other parameter combination has achieved better results. This is most likely due to the nature of the dataset and the constrained field of view of the laser, as it happens for the secondary survey, the laser mostly sees the ground therefore the degradation of ICP-based estimators. In a future work, the cylindrical 3D laser will be replaced by a hemispherical laser with a more suitable field of view.

\subsection{Qualitative analysis}

The increasing availability and affordability of 3D lasers reverberate in the demand for map registration techniques. In that context and given the dependability of the suggested system on laser-inferred odometry, we study the performance of ICP in natural environment, one of the

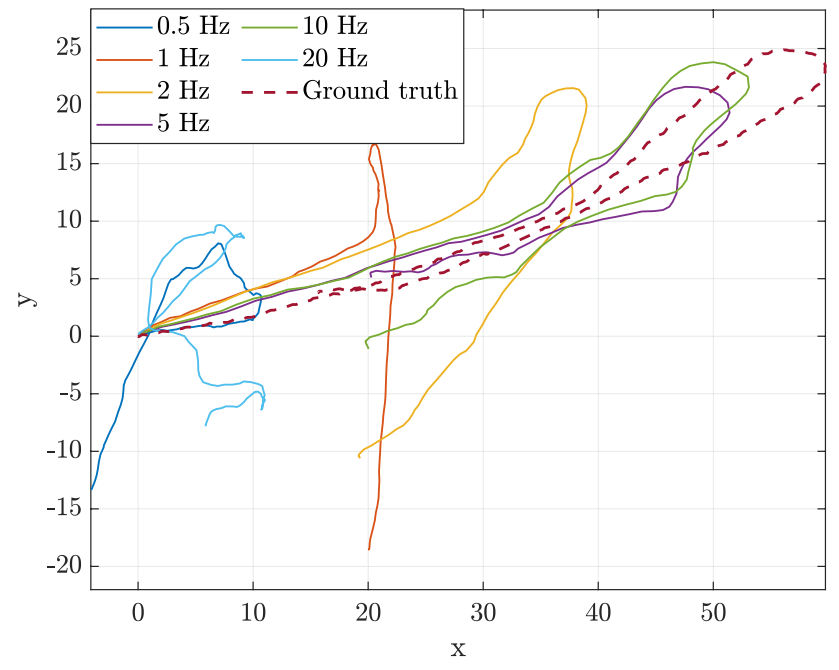

Fig. 10 Comparison between laser-based ICP and ground truth trajectories, at different data rates
Table 2 Accuracy metrics for ICP at different data rates

\begin{tabular}{llcl}
\hline Frequency $(\mathrm{Hz})$ & $\begin{array}{l}\text { Alignment RMS } \\
(\mathrm{m})\end{array}$ & Frequency $(\mathrm{Hz})$ & $\begin{array}{l}\text { Align- } \\
\text { ment } \\
\text { RMS }(\mathrm{m})\end{array}$ \\
\hline 0.5 & 33.57 & 5 & 4.43 \\
1 & 21.60 & 10 & 3.88 \\
2 & 11.37 & 20 & 32.91 \\
\hline
\end{tabular}

most used registration algorithms for laser scan registration. The initial approach was to evaluate the usability, in natural environment, of the state-of-the-art in ICP registration techniques such as TEASER++ [28]. The latter was evaluated by randomly sampling nearest neighbors for correspondence matching, but was found to be largely unreliable. The experiment was subsequently modified to take advantage of 3DSmoothNet [11] for correspondence matching, yet with no discernible performance improvement.

The performance of classical ICP i.e., with nearest neighbor correspondences was found to be easily affected by the rate at which laser scans are matched. To further elaborate on the latter hypothesis, we set the lidar operating at its maximum frequency $(20 \mathrm{~Hz})$ and then down sample the data, resulting in the plot shown in Fig. 10 and the metrics shown in Table 2. Still in the same table, it is shown that increasing the data rate increases the performance of ICP in comparison with the ground truth, yet to a certain limit after which the performance is severely degraded. Presumably, the performance degradation at the maximum frequency of $20 \mathrm{~Hz}$ is due to the relatively low speed $(<1$ $\mathrm{m} / \mathrm{s}$ ) of the person carrying the sensor suite, making small pose differentials estimated by ICP in between consecutive scans to be overshadowed by the noise surrounding the estimation.

The integration of GPS measurements into factor graph architectures is not a trivial task, due to non-Gaussian noise contamination of position estimates [18]. GPS priors, however, do have the ability to globally constrain factor graphs and therefore eliminate accumulating drift from odometry. A GPS prior could have been implemented either:

- by imposing a loose, frequent constraint i.e., a prior on every node with a relatively high variance $(>15 \mathrm{~m})$

- by imposing a tight, sparse constraint i.e., a prior every $d_{\text {gps }}$ nodes with a small variance $(<7 \mathrm{~m})$

Figure 9 shows the trajectory alignment, before and after loop closure. Notice in Fig. $9 \mathrm{~b}$ how loop closure eliminates accumulating drift in the overlapping part, although 
Fig. 11 Comparison of the effect of local reconstruction with map alignment as discussed in Sect. 3.4.1.. The above figure shows an isometric view of a scene consisting of trees and a house on the top right corner

Fig. 12 Laser-generated point cloud, showing the same scene as a colorless point cloud and a colored mesh representation

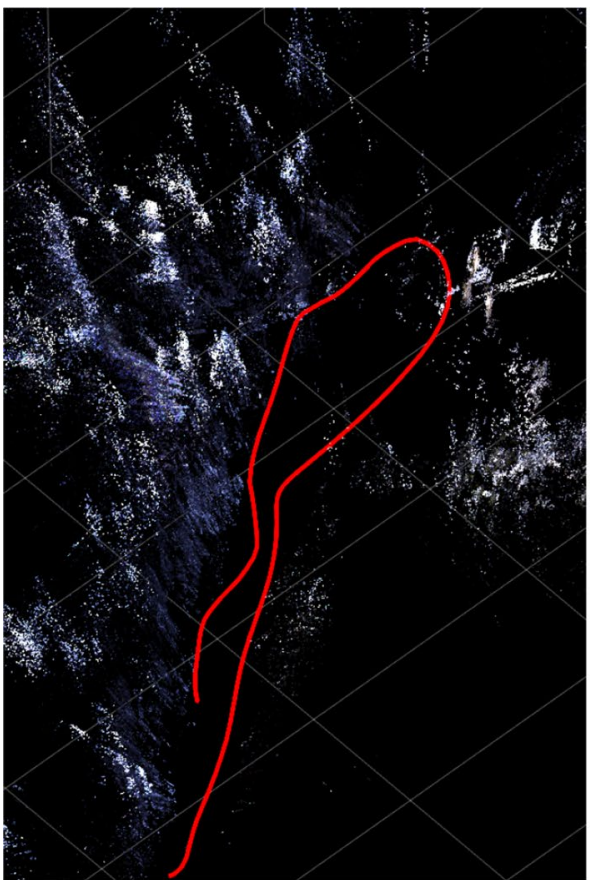

(a) $3 \mathrm{D}$ reconstruction using the optimized pose with local reconstruction and map alignment, i.e., $\mathcal{M}_{f}$

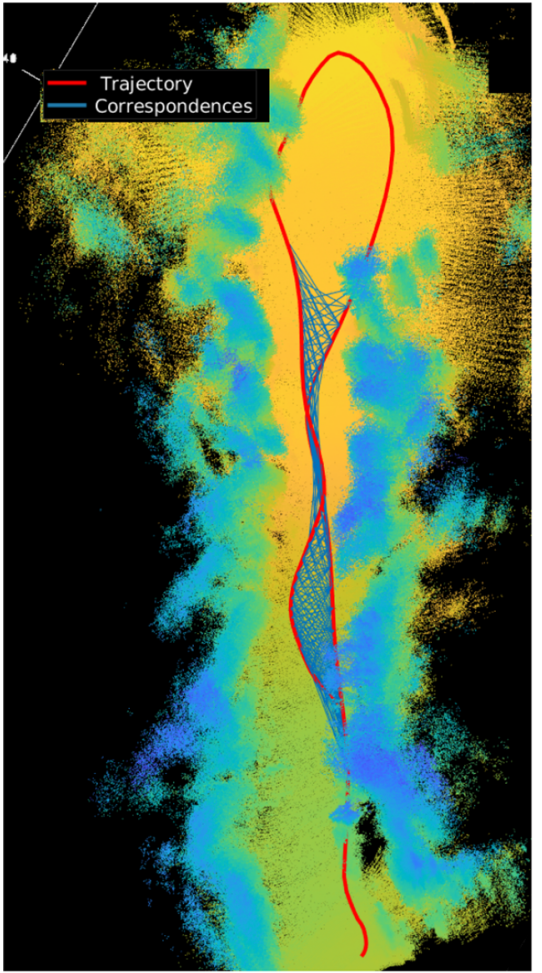

(a) Laser point cloud reconstructed using the interpolated pose from factor graph, showing a trail between 2 columns of fir trees. The blue lines are drawn using the correspondence map ( Section 3.6.1).

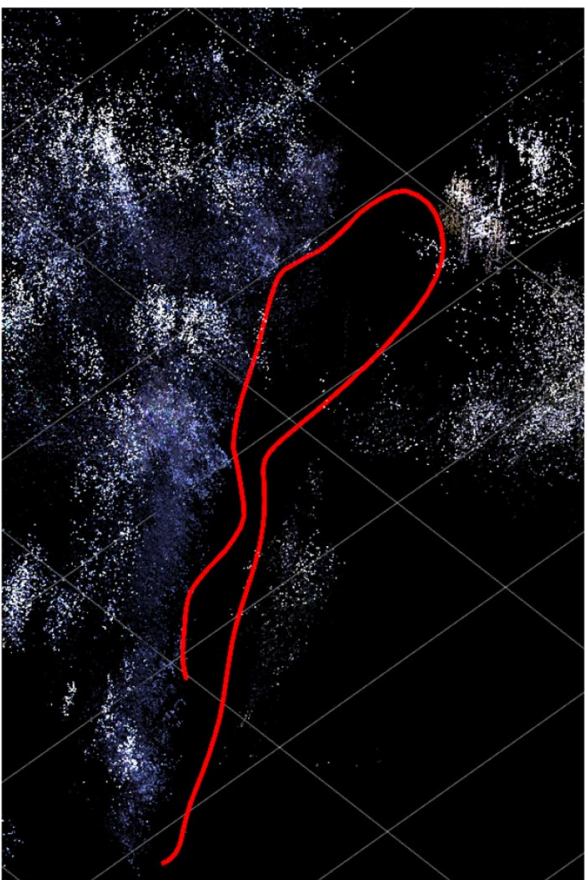

(b) Coarse map using the optimized pose without local reconstruction and map alignment, i.e., $\mathcal{M}_{c}$

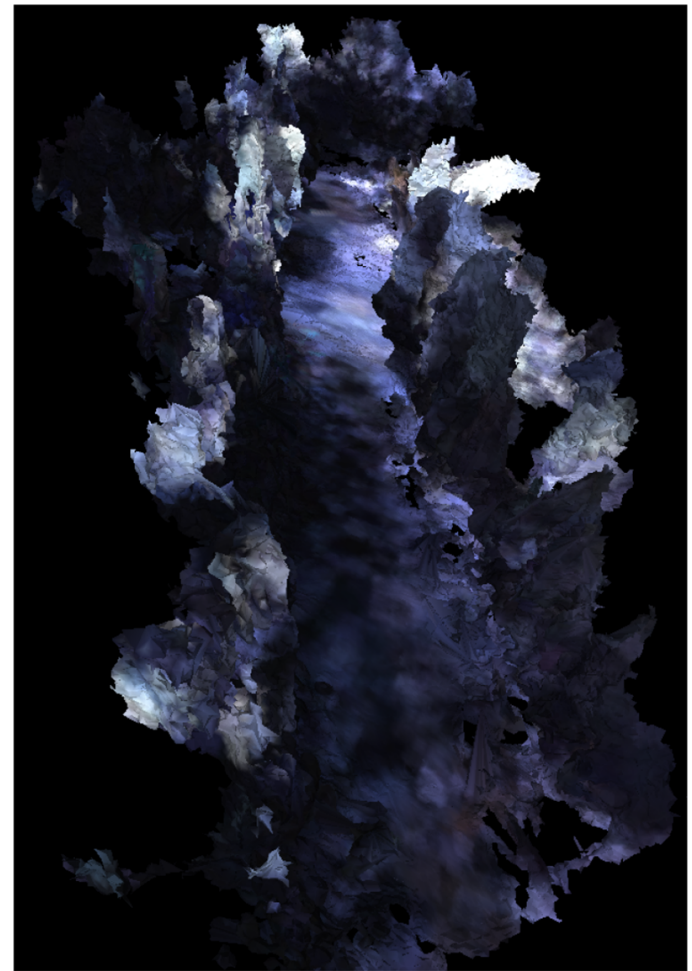

(b) Laser-generated point cloud, colored by averaging the color information of nearest neighbors in the sparse point clouds, produced from visual odometry, such as the ones shown if Figure 11. The point cloud was also meshed using the Las Vegas reconstruction toolkit.(video) 
it has little effect on the overall outcome in none-overlapping parts.

Since point cloud perception is not trivial, and since surface reconstruction improves human perception such as when displaying the maps in virtual reality, we used the Las Vegas reconstruction toolkit [24] in order to mesh the point cloud shown in Fig. 12b. Since all maps are represented in the same global frame, the colors in the latter figure have been inferred by averaging the red, green and blue channels of the 10 nearest neighbor points in the corresponding visual map, the latter generated by the center camera on the sensor suite (Fig. 6b). The lighting discrepancy is caused by varying lighting conditions, and the available shade at the moment when the data was recorded.

Although human perception of sparse point clouds can be difficult and especially in unstructured scenes, the effect of the visual reconstruction scheme suggested in Sect. 3.4.1) is nevertheless discernible in Fig. 11. Comparing Figs. $11 \mathrm{a}$ and $\mathrm{b}$, notice how noisy global reconstruction is (Fig. 11b), compared to the left-side image that uses local piece wise reconstruction with global alignment.

Unlike monocular cameras, laser-generated point clouds are dense and therefore more desirable and aesthetic. Figure 12a shows the top view of a laser-generated dense map with loop closure correspondences generated as previously discussed in Sect. 3.6.1. Still in the same figure, notice how corresponding point couples fit well within the lidar range i.e., the ensuing point cloud from local map reconstruction is guaranteed to overlap in the vicinity of the correspondences, as previously discussed in Sect. 3.6.1.

A video showing the colored point cloud being manipulated is available here.

\section{Conclusion}

We have presented a mapping framework in an extremely challenging unstructured environment, capable of integrating monocular cameras, lidars and inertial measurements with loop closure. It has been demonstrated that the presented map integration scheme is robust and results in human-readable virtual depictions of natural environment elements, using a wearable sensor suite. The importance of this achievement is of great value for scientists looking to detect changes in natural environment. The advantages of the proposed method lies in its robustness, since the state-of-the-art in visual mapping such as DSO, and the state-of-the-art in laser mapping such as ICP, cannot survive the full length of the survey without catastrophic failure. Another advantage is the flexibility of adding as many exterioceptive sensors as needed, creating a multi-layered map. For example, a near-infrared mapping layer can be added using a suitable camera, a technique that is often used by environmentalists to characterize plant type and growth. In dense forests, by filtering laser points to include only the points in a cone above the sensor suite, our method can be used to generate special types of maps, that reflect the canopy density and sky openness. The latter can be used to estimate light availability for certain plant species. Using a factor graph architecture, it is also possible to add a temporal layer, to compare maps taken at two different points in time. This approach will be addressed in a future work.

In this work, It has been shown that techniques such as ICP are of considerable utility, and can be adapted to challenging natural environment applications, for the purpose of local map alignment as well as loop closure. It has been also shown that factor graphs, when correctly manipulated, are suitable for refining pose estimates from different observations as well as to scale unitless estimates, generated using monocular techniques.

The proposed method is however limited to the availability of extrinsic parameters, which in this paper, is extracted from the CAD model of the sensor suite. Other limitations include the ability to systematically detect the failure of visual SLAM algorithms, to prevent local discontinuities in the factor graph setup. Other practical limitations for data acquisition include battery life, which is severely reduced in cold weather.

This work however exposed a considerable lack of mapping techniques suitable for natural environment applications, as well as the need to develop descriptors for 3D point clouds, and highlights the dependence of factor graphs to the non-trivial choice of uncertainty. Future work will include semantic mapping as well as temporal alignment of maps, a great interest for biologists and environmentalists who seek to detect changes in the natural environment over a long period of time. Finally, we aim to improve topological understanding of natural environment point clouds by further exploration of meshing techniques and therefore improve human perception of natural environment elements such as trees.

Funding This work was funded in part by the Grand-Est Region, the Zone Ateliers Moselle, as well as the Rhine-Meuse Water Agency in France.

Availability of data and material The dataset will be made public upon the maturity of a cross-seasonal dataset.

\section{Declaration}

Conflict of interest On behalf of all authors, the corresponding author states that there is no conflict of interest. 
Open Access This article is licensed under a Creative Commons Attribution 4.0 International License, which permits use, sharing, adaptation, distribution and reproduction in any medium or format, as long as you give appropriate credit to the original author(s) and the source, provide a link to the Creative Commons licence, and indicate if changes were made. The images or other third party material in this article are included in the article's Creative Commons licence, unless indicated otherwise in a credit line to the material. If material is not included in the article's Creative Commons licence and your intended use is not permitted by statutory regulation or exceeds the permitted use, you will need to obtain permission directly from the copyright holder. To view a copy of this licence, visit http://creativecommons. org/licenses/by/4.0/.

\section{References}

1. Babin $P$, Dandurand $P$, Kubelka $V$, Giguère $P$, Pomerleau $F$ (2019) Large-scale 3D mapping of subarctic forests. In: Proceedings of the conference on field and service robotics (FSR). Springer Tracts in Advanced Robotics

2. Besl PJ, McKay ND (1992) A method for registration of 3-D shapes. IEEE Trans Pattern Anal Mach Intell 14(2): 239-256

3. Biber $P$, Strasser $W(2003)$ The normal distributions transform: a new approach to laser scan matching. In: Proceedings 2003 IEEE/RSJ International Conference on Intelligent Robots and Systems (IROS 2003) (Cat. No.03CH37453), Las Vegas, NV, USA, vol 3, pp 2743-2748

4. Bosse M, Zlot R, Flick P (2012) Zebedee: design of a springmounted 3-D range sensor with application to mobile mapping. IEEE Trans Robotics 28(5):1104-1119

5. Cadena C, Carlone L, Carrillo H, Latif Y, Scaramuzza D, Neira J, Reid I, Leonard JJ (2016) Past, present, and future of simultaneous localization and mapping: Toward the robust-perception age. IEEE Trans Robotics 32(6):1309-1332

6. Chahine G, Pradalier C (2018) Survey of monocular slam algorithms in natural environments. In: 2018 15th conference on computer and robot vision (CRV), pp 345-352

7. Chahine G, Pradalier C (2019) Laser-supported monocular visual tracking for natural environments. In: 2019 19th international conference on advanced robotics (ICAR), pp 801-806

8. Chang CC, Chiang H (2003) Three-dimensional image reconstructions of complex objects by an abrasive computed tomography apparatus. Int J Adv Manufact Technol 22:708-712

9. Dellaert F, Kaess M (2006) Square root sam: simultaneous localization and mapping via square root information smoothing. Int J Robotics Res 25(12):1181-1203

10. Engel J, Koltun V, Cremers D (2017) Direct sparse odometry. IEEE Trans Pattern Anal Mach Intell 40(3):611-625

11. Gojcic Z, Zhou C, Wegner JD, Andreas W (2019) The perfect match: 3D point cloud matching with smoothed densities. In: International conference on computer vision and pattern recognition (CVPR)

12. Gravina R, Alinia P, Ghasemzadeh H, Fortino G (2017) Multisensor fusion in body sensor networks: state-of-the-art and research challenges. Inform Fusion 35:68-80

13. Hall DL, Llinas J (1997) An introduction to multisensor data fusion. Proc IEEE 85(1):6-23
14. Huck T, Westenberger A, Fritzsche M, Schwarz T, Dietmayer K (2011) Precise timestamping and temporal synchronization in multi-sensor fusion. In: 2011 IEEE intelligent vehicles symposium (IV), pp 242-247

15. Kato A, Moskal LM, Schiess P, Swanson ME, Calhoun D, Stuetzle W (2009) Capturing tree crown formation through implicit surface reconstruction using airborne lidar data. Remote Sens Environ 113(6):1148-1162

16. Koide K, Miura J, Menegatti E (2019) A portable three-dimensional lidar-based system for long-term and wide-area people behavior measurement. Int J Adv Robot Syst. https://doi.org/10. $1177 / 1729881419841532$

17. Kümmerle R, Grisetti G, Strasdat H, Konolige K, Burgard W (2011) g 2 o: a general framework for graph optimization. In: 2011 IEEE international conference on robotics and automation. IEEE, pp 3607-3613

18. Liu L, Amin MG (2008) Performance analysis of gps receivers in non-gaussian noise incorporating precorrelation filter and sampling rate. IEEE Trans Signal Process 56(3):990-1004

19. Pierzchała $M$, Giguère $P$, Astrup $R$ (2018) Mapping forests using an unmanned ground vehicle with 3D LiDAR and graph-SLAM. Comput Electron Agric 145:217-225

20. Pomerleau F, Colas F, Siegwart R, Magnenat S (2013) Comparing ICP variants on real-world data sets: Open-source library and experimental protocol. Autonomous Robots 34(3):133-148

21. Pomerleau F, Magnenat S, Colas F, Liu M, Siegwart R (2011) Tracking a depth camera: parameter exploration for fast icp. In: 2011 IEEE/RSJ international conference on intelligent robots and systems, pp 3824-3829

22. Shoemake $K$ (1985) Animating rotation with quaternion curves. SIGGRAPH Comput Graph 19(3):245-254

23. Tremblay JF, Béland $M$, Pomerleau $F$, Gagnon $R$, Giguère $P$ (2019) Automatic 3D mapping for tree diameter measurements in inventory operations. In: Proceedings of the conference on field and service robotics (FSR). Springer Tracts in Advanced Robotics

24. Wiemann T, Nuechter A, Hertzberg J (2012) A toolkit for automatic generation of polygonal maps-las vegas reconstruction. In: ROBOTIK 2012; 7th German conference on robotics, pp 1-6

25. Wu W, Wang Z, Li Z, Liu W, Fuxin L (2019) Pointpwc-net: a coarseto-fine network for supervised and self-supervised scene flow estimation on 3D point clouds. arXiv preprint arXiv:1911.12408

26. Wu X, Pradalier C (2019) Illumination robust monocular direct visual odometry for outdoor environment mapping. In: 2019 International conference on robotics and automation (ICRA), pp 2392-2398

27. Wu X, Vela P, Pradalier C (2019) Robust monocular edge visual odometry through coarse-to-fine data association. In: 2020 IEEE/RSJ International Conference on Intelligent Robots and Systems (IROS), Las Vegas, NV, USA, pp 4923-4929

28. Yang H, Shi J, Carlone L (2020) Teaser: fast and certifiable point cloud registration. arXiv:abs/2001.07715

29. Zhang J, Singh S (2015) LOAM: Lidar odometry and mapping in real-time. IEEE Trans Robotics 32(7):141-148

Publisher's Note Springer Nature remains neutral with regard to jurisdictional claims in published maps and institutional affiliations. 